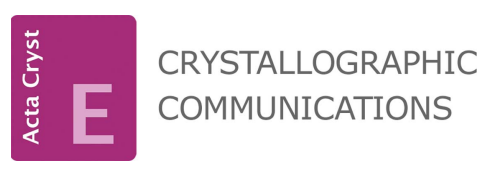

ISSN 2056-9890

Received 14 July 2021

Accepted 18 July 2021

Edited by B. Therrien, University of Neuchâtel, Switzerland

Keywords: crystal structure; alkyne; dihydroquinoline; hydrogen bond; Hirshfeld surface analysis.

CCDC reference: 2097267

Supporting information: this article has supporting information at journals.iucr.org/e

\section{Crystal structure, Hirshfeld surface analysis and density functional theory study of benzyl 2-oxo-1- (prop-2-yn-1-yl)-1,2-dihydroquinoline-4-carboxyl- ate}

\author{
Younos Bouzian, ${ }^{a}$ Karim Chkirate, ${ }^{a}$ Joel T. Mague, ${ }^{b}$ Fares Hezam Al-Ostoot, ${ }^{{ }^{*} *}$ \\ Noureddine Hammou Ahabchane ${ }^{\mathrm{a}}$ and El Mokhtar Essassi ${ }^{\mathrm{a}}$
}

\begin{abstract}
a'Laboratory of Heterocyclic Organic Chemistry URAC 21, Pharmacochemistry Competence Center, Av. Ibn Battouta, BP 1014, Faculty of Sciences, Mohammed V University, Rabat, Morocco, bepartment of Chemistry, Tulane University, New Orleans, LA 70118, USA, and ' Department of Biochemistry, Faculty of Education \& Science, Al-Baydha University, Yemen. *Correspondence e-mail: faresalostoot@gmail.com
\end{abstract}

The title molecule, $\mathrm{C}_{20} \mathrm{H}_{15} \mathrm{NO}_{3}$, adopts a $Z$-shaped conformation with the carboxyl group nearly coplanar with the dihydroquinoline unit. In the crystal, corrugated layers are formed by $\mathrm{C}-\mathrm{H} \cdots \mathrm{O}$ hydrogen bonds and are stacked by $\mathrm{C}-\mathrm{H} \cdots \pi$ (ring) interactions. Hirshfeld surface analysis indicates that the most important contributions to the crystal packing are from $\mathrm{H} \cdots \mathrm{H}(43.3 \%), \mathrm{H} \cdots \mathrm{C} /$ $\mathrm{C} \cdot \mathrm{H}(26.6 \%)$ and $\mathrm{H} \cdots \mathrm{O} / \mathrm{O} \cdots \mathrm{H}(16.3 \%)$ interactions. The optimized structure calculated using density functional theory at the B3LYP/ 6-311 G(d,p) level is compared with the experimentally determined structure in the solid state. The calculated HOMO-LUMO energy gap is $4.0319 \mathrm{eV}$.

\section{Chemical context}

Nitrogen-based structures have attracted increased attention in recent years because of their interesting properties in structural and inorganic chemistry (Chkirate et al., 2019, $2020 a, b, 2021)$. The family of quinolines, particularly those containing the 2-oxoquinoline moiety, is important in medicinal chemistry because of their wide range of pharmacological applications including as potential anticancer agents (Fang et al., 2021), anti-proliferative agents (Banu et al., 2017) and as potent modulators of ABCB1-related drug resistance of mouse T-lymphoma cells (Filali Baba et al., 2020). In particular, 2-oxoquinoline-4-carboxylate derivatives are active antioxidants (Filali Baba et al., 2019). Given the wide range of therapeutic applications for such compounds, and in a continuation of the work already carried out on the synthesis of compounds resulting from quinolin-2-one (Bouzian et al., 2020), a similar approach gave the title compound, benzyl 2-oxo-1-(prop-2-yn-1-yl)-1,2-dihydroquinoline-4-carboxylate, (I). Besides the synthesis, we also report the molecular and crystalline structures along with a Hirshfeld surface analysis and a density functional theory computational calculation carried out at the B3LYP/6- 311 G(d,p) level.<smiles>C#CCn1c(=O)cc(C(=O)OCc2ccccc2)c2ccccc21</smiles> 
Table 1

Hydrogen-bond geometry $\left(\AA,^{\circ}\right)$.

$\mathrm{Cg} 2$ and $\mathrm{Cg} 3$ are the centroids of the $\mathrm{C} 1-\mathrm{C} 6$ and $\mathrm{C} 15-\mathrm{C} 20$ benzene rings, respectively.

\begin{tabular}{lllll}
\hline$D-\mathrm{H} \cdots A$ & $D-\mathrm{H}$ & $\mathrm{H} \cdots A$ & $D \cdots A$ & $D-\mathrm{H} \cdots A$ \\
\hline $\mathrm{C} 2-\mathrm{H} 2 \cdots C g 3^{\mathrm{i}}$ & 0.95 & 2.94 & $3.8206(10)$ & 154 \\
$\mathrm{C} 4-\mathrm{H} 4 \cdots \mathrm{O} 2^{\mathrm{ii}}$ & 0.95 & 2.57 & $3.4846(11)$ & 162 \\
$\mathrm{C} 5-\mathrm{H} 5 \cdots \mathrm{O} 2$ & 0.95 & 2.23 & $2.8917(11)$ & 126 \\
$\mathrm{C} 12-\mathrm{H} 12 \cdots \mathrm{O} 1^{\mathrm{iii}}$ & 0.95 & 2.25 & $3.1463(14)$ & 157 \\
$\mathrm{C} 14-\mathrm{H} 14 A \cdots C g 2^{\text {iv }}$ & 0.99 & 2.65 & $3.4652(9)$ & 140 \\
$\mathrm{C} 16-\mathrm{H} 16 \cdots \mathrm{O} 1^{\mathrm{v}}$ & 0.95 & 2.50 & $3.3443(12)$ & 148 \\
\hline
\end{tabular}

Symmetry codes: (i) $-x+1,-y+1,-z+1$; (ii) $\quad-x,-y+1,-z+1$; $\quad$ (iii) $-x+\frac{3}{2}, y+\frac{1}{2},-z+\frac{1}{2}$; (iv) $-x+\frac{1}{2}, y-\frac{1}{2},-z+\frac{1}{2} ;$ (v) $-x+\frac{3}{2}, y-\frac{1}{2},-z+\frac{1}{2}$.

\section{Structural commentary}

The molecule adopts a Z-shaped conformation with the propynyl and benzyl substituents projecting from opposite sides of the mean plane of the dihydroquinoline moiety. This moiety is planar to within 0.0340 (6) $\AA$ (r.m.s. deviation = $0.0164)$ with $\mathrm{N} 1$ and $\mathrm{C} 9$ being, respectively, 0.0340 (6) and -0.0279 (7) $\AA$ from the mean plane, resulting in a slight twist at this location. The carboxyl group is nearly coplanar with the dihydroquinoline as seen from the $1.04(5)^{\circ}$ dihedral angle between the plane defined by $\mathrm{C} 7 / \mathrm{C} 13 / \mathrm{O} 2 / \mathrm{O} 3$ and that of the dihydroquinoline $(\mathrm{C} 1-\mathrm{C} 9 / \mathrm{N} 1 / \mathrm{O} 1)$. This is likely due, in part, to the intramolecular $\mathrm{C} 5-\mathrm{H} 5 \cdots \mathrm{O} 2$ interaction (Table 1 and Fig. 1). The propynyl substituent is rotated out of the mean plane of the dihydroquinoline moiety by $80.88(3)^{\circ}$. The plane of the $\mathrm{C} 15-\mathrm{C} 20$ ring is inclined to that of the dihydroquinoline by $68.47(2)^{\circ}$.

\section{Supramolecular features}

In the crystal, $\mathrm{C} 12-\mathrm{H} 12 \cdots \mathrm{O} 1$ and $\mathrm{C} 16-\mathrm{H} 16 \cdots \mathrm{O} 1$ hydrogen bonds (Table 1) link the molecules into zigzag chains extending along the $b$-axis direction, which are connected by inversion-related pairs of $\mathrm{C} 4-\mathrm{H} 4 \cdots \mathrm{O} 2$ hydrogen bonds (Table 1) into corrugated layers parallel to the (103) plane

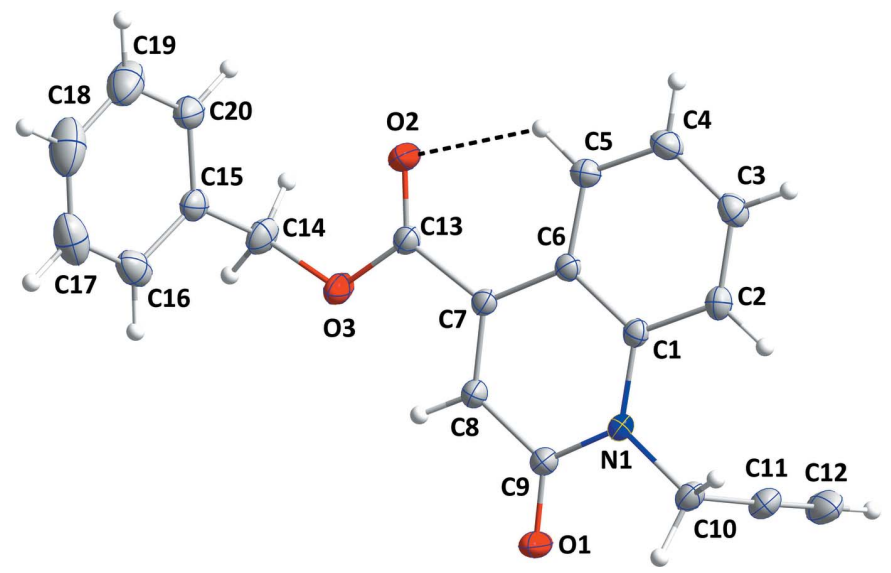

Figure 1

The title molecule with labeling scheme and $50 \%$ probability ellipsoids. The intramolecular hydrogen bond is depicted by a dashed line.

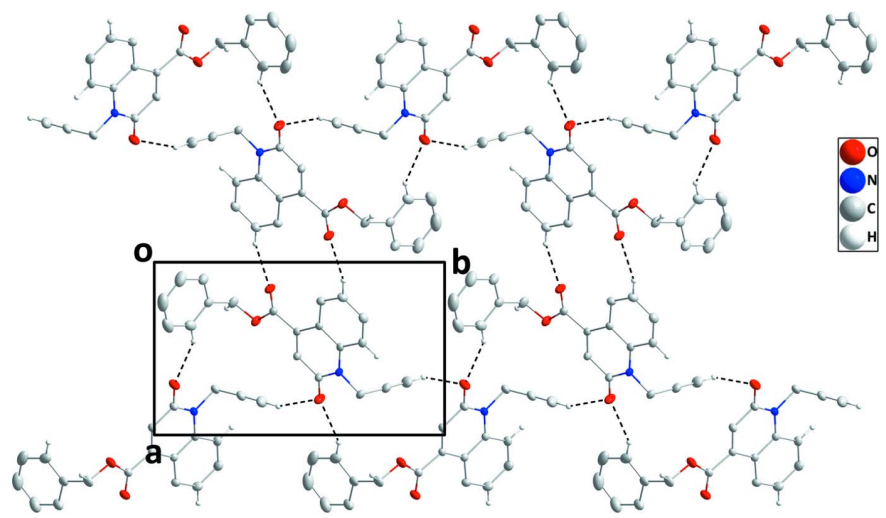

Figure 2

A portion of one layer viewed along the $c$ axis with $\mathrm{C}-\mathrm{H} \cdots \mathrm{O}$ hydrogen bonds depicted by dashed lines.

(Fig. 2). The layers are stacked along the normal to (103) with $\mathrm{C} 2-\mathrm{H} 2 \cdots \mathrm{Cg} 3$ and $\mathrm{C} 14-\mathrm{H} 14 A \cdots C g 2$ interactions (Table 1 and Fig. 3).

\section{Hirshfeld surface analysis}

The CrystalExplorer program (Turner et al., 2017) was used to investigate and visualize further the intermolecular interactions of (I). The Hirshfeld surface plotted over $d_{\text {norm }}$ in the range -0.3677 to 1.3896 a.u. is shown in Fig. $4 a$. The electrostatic potential using the STO-3G basis set at the HartreeFock level of theory and mapped on the Hirshfeld surface over the range of \pm 0.05 a.u. clearly shows the positions of close intermolecular contacts in the compound (Fig. 4b). The posi-

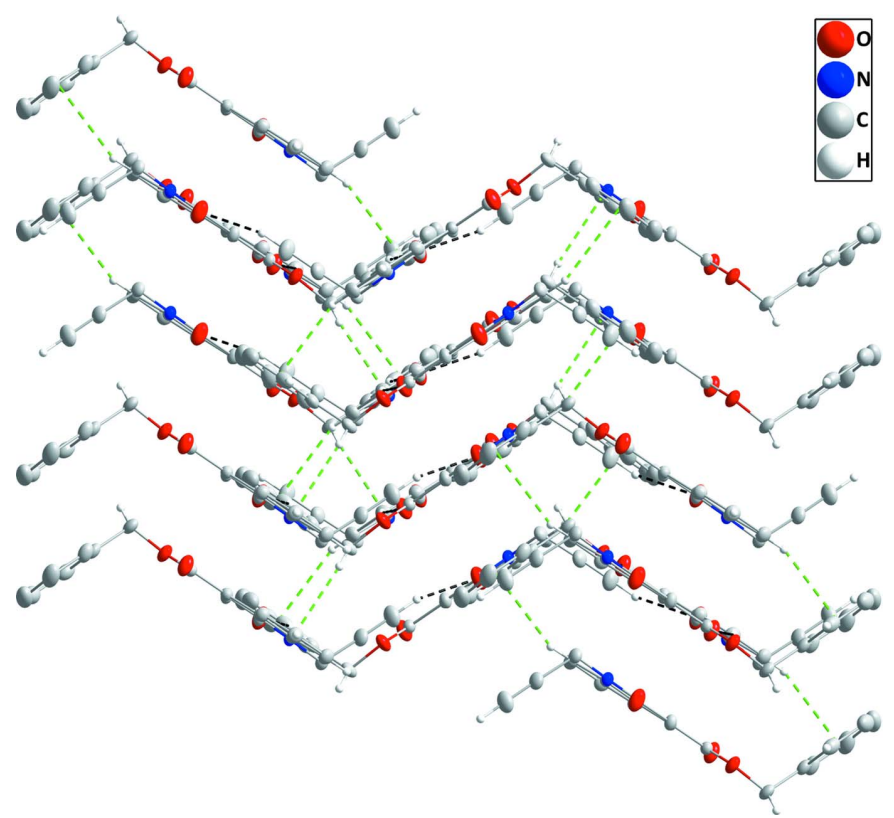

Figure 3

Packing viewed parallel to (103) with the $b$ axis horizontal and running from left to right. $\mathrm{C}-\mathrm{H} \cdots \mathrm{O}$ hydrogen bonds and $\mathrm{C}-\mathrm{H} \cdots \pi$ (ring) interactions are depicted, respectively, by black and green dashed lines. 


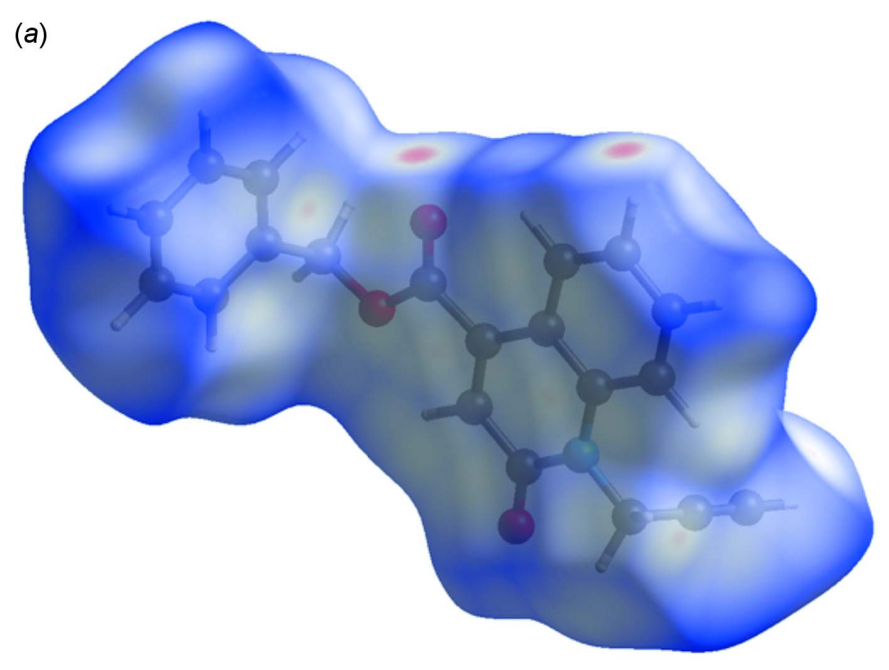

(b)

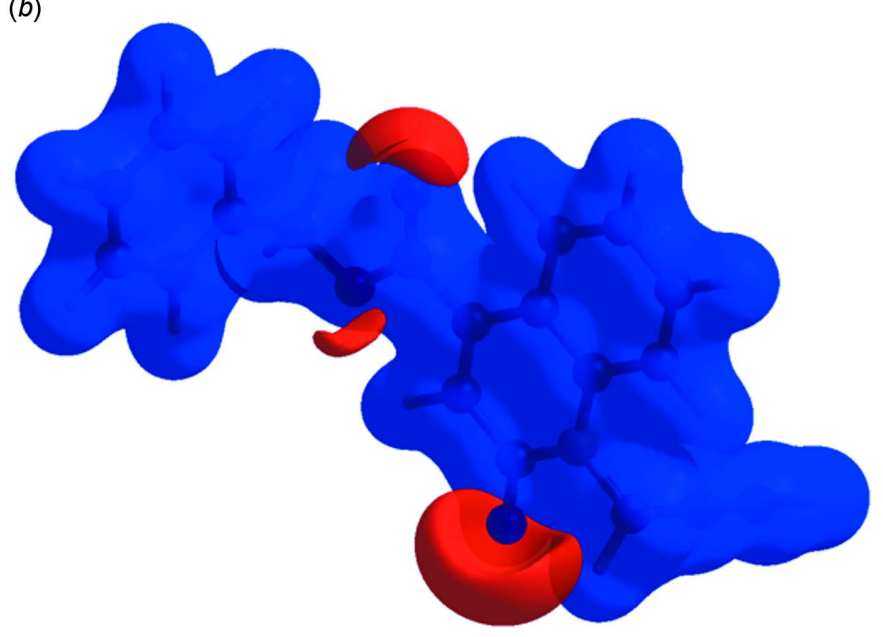

Figure 4

(a) View of the three-dimensional Hirshfeld surface of the title compound, plotted over $d_{\text {norm }}$ in the range of -0.3677 to 1.3896 a.u. (b) View of the three-dimensional Hirshfeld surface of the title compound plotted over electrostatic potential energy in the range -0.0500 to 0.0500 a.u. using the STO-3 G basis set at the Hartree-Fock level of theory.

tive electrostatic potential (blue region) over the surface indicates hydrogen-donor potential, whereas the hydrogenbond acceptors are represented by negative electrostatic potential (red region).

The overall two-dimensional fingerprint plot (McKinnon et $a l ., 2007)$ is shown in Fig. $5 a$, while those delineated into $\mathrm{H} \cdots \mathrm{H}, \mathrm{H} \cdots \mathrm{C} / \mathrm{C} \cdots \mathrm{H}, \mathrm{H} \cdots \mathrm{O} / \mathrm{O} \cdots \mathrm{H}, \mathrm{C} \cdots \mathrm{C}, \mathrm{O} \cdots \mathrm{C} / \mathrm{C} \cdots \mathrm{O}$, $\mathrm{H} \cdots \mathrm{N} / \mathrm{N} \cdots \mathrm{H}, \mathrm{N} \cdots \mathrm{C} / \mathrm{C} \cdots \mathrm{N}$ and $\mathrm{N} \cdots \mathrm{O} / \mathrm{O} \cdots \mathrm{N}$ contacts are illustrated in Fig. $5 b-i$, respectively, together with their relative contributions to the Hirshfeld surface (HS). The most important interaction is $\mathrm{H} \cdots \mathrm{H}$, contributing $43.3 \%$ to the overall crystal packing, which is reflected in Fig. $5 b$ as widely scattered points of high density due to the large hydrogen content of the molecule, with its tip at $d_{\mathrm{e}}=d_{\mathrm{i}}=1.19 \AA$. In the presence of $\mathrm{C}-\mathrm{H}$ interactions, the pair of characteristic wings in the fingerprint plot delineated into $\mathrm{H} \cdots \mathrm{C} / \mathrm{C} \cdots \mathrm{H}$ contacts ( $26.6 \%$ contribution to the HS, Fig. $5 c$ ) has tips at $d_{\mathrm{e}}+d_{\mathrm{i}}=$ $3.07 \AA$. The pair of scattered points of spikes in the fingerprint plot delineated into $\mathrm{H} \cdots \mathrm{O} / \mathrm{O} \cdots \mathrm{H}$ contacts (Fig. $5 d, 16.3 \%$ )
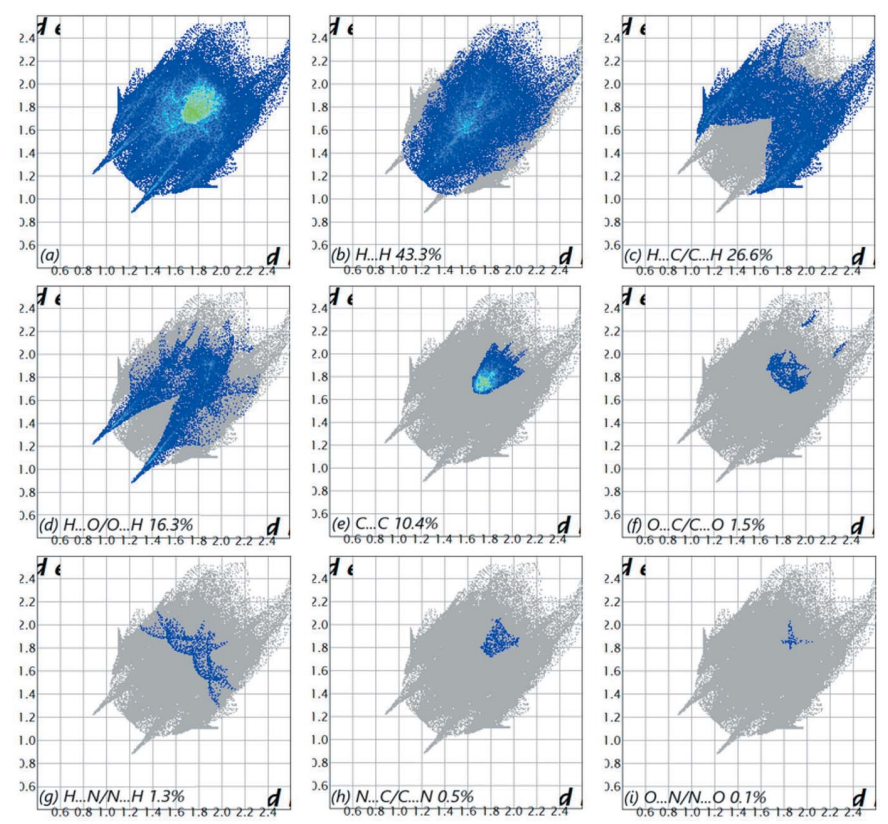

Figure 5

The full two-dimensional fingerprint plots for the title compound, showing (a) all interactions, and delineated into $(b) \mathrm{H} \cdots \mathrm{H},(c) \mathrm{H} \cdots \mathrm{C} /$ $\mathrm{C} \cdots \mathrm{H},(d) \mathrm{H} \cdots \mathrm{O} / \mathrm{O} \cdots \mathrm{H},(e) \mathrm{C} \cdots \mathrm{C},(f) \mathrm{O} \cdots \mathrm{C} / \mathrm{C} \cdots \mathrm{O},(g) \mathrm{H} \cdots \mathrm{N} / \mathrm{N} \cdots \mathrm{H}$, (h) $\mathrm{N} \cdots \mathrm{C} / \mathrm{C} \cdots \mathrm{N}$ and (i) $\mathrm{N} \cdots \mathrm{O} / \mathrm{O} \cdots \mathrm{N}$ interactions. $d_{i}$ and $d_{e}$ values are the closest internal and external distances (in $\AA$ ) from given points on the Hirshfeld surface.

have tips at $d_{\mathrm{e}}+d_{\mathrm{i}}=2.08 \AA$. The $\mathrm{C} \cdots \mathrm{C}$ contacts (Fig. 5e, $10.4 \%$ ) have tips at $d_{\mathrm{e}}+d_{\mathrm{i}}=3.34 \AA$. The $\mathrm{O} \cdots \mathrm{C} / \mathrm{C} \cdots \mathrm{O}$ contacts, Fig. $5 f$, contribute $1.5 \%$ to the HS and appear as a pair of scattered points of spikes with tips at $d_{\mathrm{e}}+d_{\mathrm{i}}=3.55 \AA$. The $\mathrm{H} \cdots \mathrm{N} / \mathrm{N} \cdots \mathrm{H}$ contacts (Fig. $5 g, 1.3 \%$ ) have tips at $d_{\mathrm{e}}+d_{\mathrm{i}}$ $=3.28 \AA$. Finally, the $\mathrm{C} \cdots \mathrm{N} / \mathrm{N} \cdots \mathrm{C}$ and $\mathrm{O} \cdots \mathrm{N} / \mathrm{N} \cdots \mathrm{O}$ contacts, Fig. $5 h-i$, contribute only $0.5 \%$ and $0.1 \%$ respectively to the HS and have a low-density distribution of points.

\section{Density Functional Theory calculations}

The structure in the gas phase of the title compound was optimized by means of density functional theory. The density functional theory calculation was performed by the hybrid B3LYP method and the 6-311 G(d,p) basis-set, which is based on Becke's model (Becke, 1993) and considers a mixture of the exact (Hartree-Fock) and density functional theory exchange utilizing the B3 functional, together with the LYP correlation functional (Lee et al., 1988). After obtaining the converged geometry, the harmonic vibrational frequencies were calculated at the same theoretical level to confirm that the number of imaginary frequencies is zero for the stationary point. Both the geometry optimization and harmonic vibrational frequency analysis of the title compound were performed with the Gaussian 09 program (Frisch et al., 2009). Theoretical and experimental results related to bond lengths and angles are in good agreement, and are summarized in Table 2. Calculated numerical values for the title compound including electronegativity $(\chi)$, hardness $(\eta)$, ionization 
Table 2

Comparison (X-ray and DFT) of selected bond lengths and angles $\left(\AA{ }^{\circ}\right)$.

\begin{tabular}{lll}
\hline & X-ray & B3LYP/6-311G(d,p) \\
\hline O1-C9 & $1.2355(10)$ & 1.223 \\
O3-C13 & $1.3375(10)$ & 1.3447 \\
N1-C9 & $1.3788(10)$ & 1.4042 \\
N1-C10 & $1.4730(10)$ & 1.4725 \\
O2-C13 & $1.2058(10)$ & 1.2092 \\
O3-C14 & $1.4588(10)$ & 1.4611 \\
N1-C1 & $1.3999(10)$ & 1.3953 \\
& & \\
C13-O3-C14 & $116.87(7)$ & 117.1258 \\
C9-N1-C10 & $115.85(6)$ & 115.6313 \\
N1-C1-C2 & $119.87(7)$ & 120.5532 \\
O1-C9-N1 & $121.42(7)$ & 121.7499 \\
N1-C9-C8 & $116.04(7)$ & 115.2168 \\
O2-C13-C7 & $125.74(7)$ & 125.0357 \\
O3-C14-C15 & $112.63(7)$ & 111.678 \\
C9-N1-C1 & $123.16(6)$ & 123.4431 \\
C1-N1-C10 & $120.93(6)$ & 120.911 \\
N1-C1-C6 & $120.08(6)$ & 120.1155 \\
O1-C9-C8 & $122.54(7)$ & 113.0317 \\
C11-C10-N1 & $111.46(7)$ & 123.6586 \\
O2-C13-O3 & $123.21(7)$ & 111.3015 \\
O3-C13-C7 & $111.05(6)$ &
\end{tabular}

potential $(I)$, dipole moment $(\mu)$, electron affinity $(A)$, electrophilicity $(\omega)$ and softness $(\sigma)$ are collated in Table 3 . The electron transition from the highest occupied molecular orbital (HOMO) to the lowest unoccupied molecular orbital (LUMO) energy level is shown in Fig. 6. The HOMO and LUMO are localized in the plane extending over the whole benzyl 2-oxo-1-(prop-2-yn-1-yl)-1,2-dihydroquinoline-4-carboxylate system. The energy band gap $\left(\Delta E=E_{\mathrm{LUMO}}-E_{\mathrm{HOMO}}\right)$ of the molecule is $4.0319 \mathrm{eV}$, and the frontier molecular orbital energies, $E_{\mathrm{HOMO}}$ and $E_{\mathrm{LUMO}}$, are -6.3166 and $-2.2847 \mathrm{eV}$, respectively.

\section{Database survey}

A search of the Cambridge Structural Database (CSD version 5.42, updated May 2021; Groom et al., 2016) with the 2-oxo-1(prop-2-yn-1-yl)-1,2-dihydroquinoline-4-carboxylate fragment yielded multiple matches. Of these, two had an alkyl substituent on $\mathrm{O} 3$ comparable to (I). The first compound (refcode OKIGAT; Hayani et al., 2021) carries an ethyl group on O3, while the second one (refcode OKIGOH; Hayani et al., 2021) carries a cyclohexyl group. The ethyl carboxylate in OKIGAT forms a dihedral angle of $-8.3(7)^{\circ}$ with the dihydroquinoline unit. In OKIGOH, the dihedral angle between the mean planes of the cyclohexyl carboxylate and dihydroquinoline rings is $37.3(8)^{\circ}$. As previously mentioned, the carboxyl group in (I) is nearly coplanar with the dihydroquinoline [dihedral angle of $1.04(5)^{\circ}$, which is approximately the same as in OKIGAT, but less tilted than in OKIGOH.

\section{Synthesis and crystallization}

A mixture of 2-oxo-1-(prop-2-yn-1-yl)-1,2-dihydroquinoline4-carboxylic acid $(0.7 \mathrm{~g}, 3 \mathrm{mmol}), \mathrm{K}_{2} \mathrm{CO}_{3}(0.51 \mathrm{~g}, 3.6 \mathrm{mmol})$, benzyl chloride $(0.76 \mathrm{ml}, 6 \mathrm{mmol})$ and tetra $n$-butylammonium
Table 3

Calculated energies.

\begin{tabular}{lr}
\hline Molecular energy & Compound (I) \\
\hline Total energy $T E(\mathrm{eV})$ & -28621.0571 \\
$E_{\mathrm{HOMO}}(\mathrm{eV})$ & -6.3166 \\
$E_{\mathrm{LUMO}}(\mathrm{eV})$ & -2.2847 \\
Gap, $\Delta E(\mathrm{eV})$ & 4.0319 \\
Dipole moment, $\mu$ (Debye) & 1.9469 \\
Ionization potential, $I(\mathrm{eV})$ & 6.3166 \\
Electron affinity, $A$ & 2.2847 \\
Electronegativity, $\chi$ & 4.3007 \\
Hardness, $\eta$ & 2.0160 \\
Electrophilicity index, $\omega$ & 4.5873 \\
Softness, $\sigma$ & 0.4960 \\
Fraction of electron transferred, $\Delta N$ & 0.6695 \\
\hline
\end{tabular}

bromide as a catalyst in DMF $(30 \mathrm{~mL})$ was stirred at room temperature for $48 \mathrm{~h}$. After removal of the salts by filtration, the solvent was evaporated under reduced pressure and the residue obtained was dissolved in dichloromethane. The organic phase was dried over $\mathrm{Na}_{2} \mathrm{SO}_{4}$ and concentrated under vacuum. The crude product obtained was purified by chro-
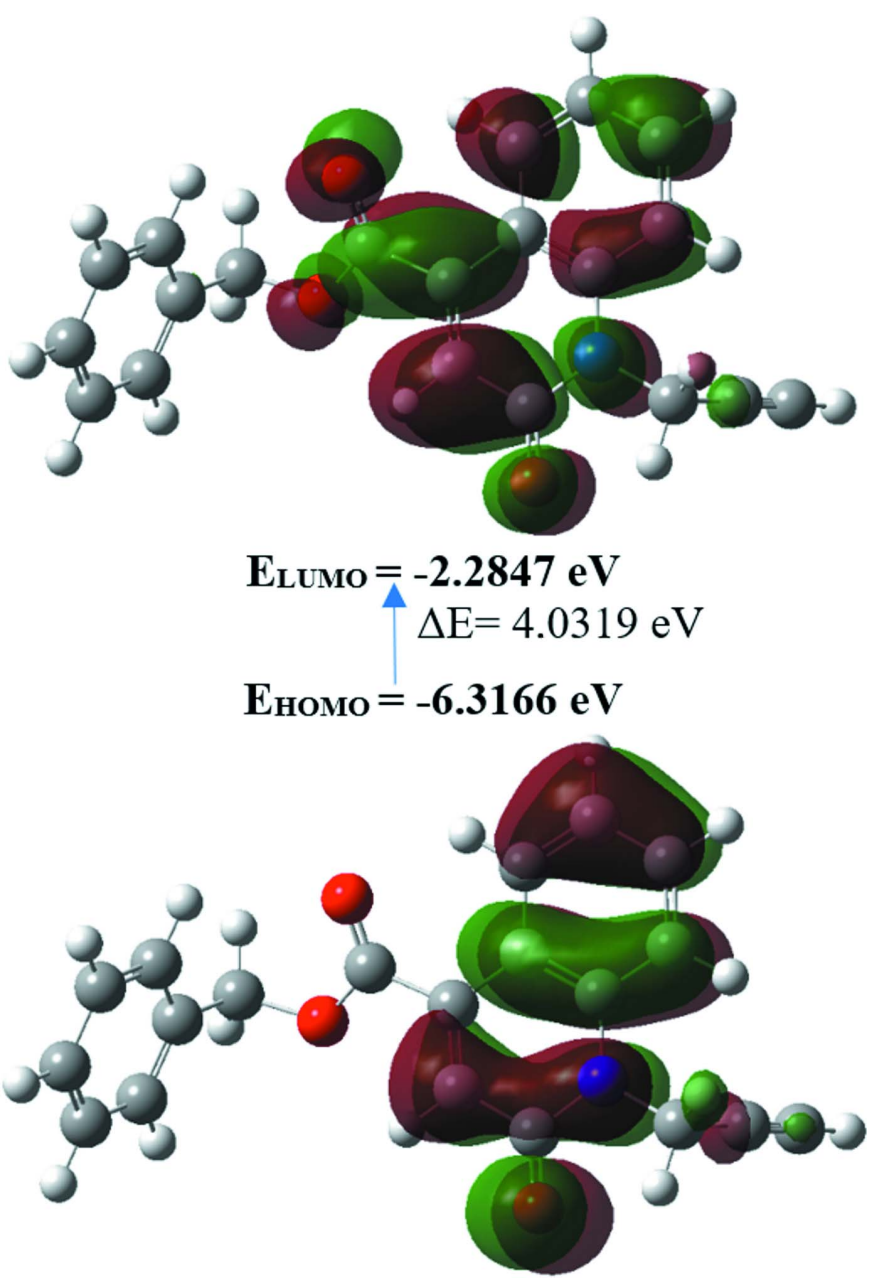

Figure 6

The energy band gap of benzyl 2-oxo-1-(prop-2-yn-1-yl)-1,2-dihydroquinoline-4-carboxylate. 
Table 4

Experimental details.

\begin{tabular}{|c|c|}
\hline \multicolumn{2}{|l|}{ Crystal data } \\
\hline Chemical formula & $\mathrm{C}_{20} \mathrm{H}_{15} \mathrm{NO}_{3}$ \\
\hline$M_{\mathrm{r}}$ & 317.33 \\
\hline Crystal system, space group & Monoclinic, $P 2_{1} / n$ \\
\hline Temperature $(\mathrm{K})$ & 150 \\
\hline$a, b, c(\AA)$ & $8.2284(3), 13.7693(4), 13.9230(4)$ \\
\hline$\beta\left(^{\circ}\right)$ & $96.155(1)$ \\
\hline$V\left(\AA^{3}\right)$ & $1568.37(9)$ \\
\hline$Z$ & 4 \\
\hline Radiation type & Мо $K \alpha$ \\
\hline$\mu\left(\mathrm{mm}^{-1}\right)$ & 0.09 \\
\hline Crystal size $(\mathrm{mm})$ & $0.44 \times 0.35 \times 0.32$ \\
\hline \multicolumn{2}{|l|}{ Data collection } \\
\hline Diffractometer & $\begin{array}{l}\text { Bruker D8 QUEST PHOTON } 3 \\
\text { diffractometer }\end{array}$ \\
\hline Absorption correction & $\begin{array}{l}\text { Numerical (SADABS; Krause et } \\
\quad \text { al., 2015) }\end{array}$ \\
\hline$T_{\min }, T_{\max }$ & $0.93,0.97$ \\
\hline $\begin{array}{l}\text { No. of measured, independent and } \\
\text { observed }[I>2 \sigma(I)] \text { reflections }\end{array}$ & $80207,6020,5304$ \\
\hline$R_{\text {int }}$ & 0.025 \\
\hline$(\sin \theta / \lambda)_{\max }\left(\AA^{-1}\right)$ & 0.774 \\
\hline \multicolumn{2}{|l|}{ Refinement } \\
\hline$R\left[F^{2}>2 \sigma\left(F^{2}\right)\right], w R\left(F^{2}\right), S$ & $0.046,0.133,1.03$ \\
\hline No. of reflections & 6020 \\
\hline No. of parameters & 217 \\
\hline $\mathrm{H}$-atom treatment & $\mathrm{H}$-atom parameters constrained \\
\hline$\Delta \rho_{\max }, \Delta \rho_{\min }\left(\mathrm{e} \AA^{-3}\right)$ & $0.46,-0.21$ \\
\hline
\end{tabular}

Computer programs: APEX3 and SAINT (Bruker, 2020), SHELXT (Sheldrick, 2015a), SHELXL2018/1 (Sheldrick, 2015b), DIAMOND (Brandenburg \& Putz, 2012) and SHELXTL (Sheldrick, 2008).

matography on a column of silica gel (eluent: hexane/ ethyl acetate: 9/1). ${ }^{1} \mathrm{H}$ NMR (300 MHz, DMSO- $\left.d_{6}\right) \delta$ ppm: $3.08(t$, $1 \mathrm{H}, \mathrm{CH} \equiv)$; $4.37\left(d, 2 \mathrm{H}, \mathrm{CH}_{2}-\mathrm{N}\right)$; $5.12\left(s, 2 \mathrm{H}, \mathrm{CH}_{2}-\mathrm{O}\right) ; 7.08-$ $8.74\left(m, 10 \mathrm{H}, \mathrm{CH}_{\text {arom }}\right) ;{ }^{13} \mathrm{C} \mathrm{NMR}\left(75 \mathrm{MHz}, \mathrm{DMSO}-d_{6}\right) \delta \mathrm{ppm}$ : $34.3\left(\mathrm{CH}_{3}-\mathrm{N}\right) ; 66.2\left(\mathrm{CH}_{2}-\mathrm{O}\right) ; 72.1(-\mathrm{C} \equiv) ; 73.2(\mathrm{CH} \equiv)$; 115.6-148.7 ( $\mathrm{CH}_{\text {arom }}$ and $\left.\mathrm{C}_{\text {quat arom }}\right) ; 162.5\left(\mathrm{C}=\mathrm{O}_{\text {quinol }}\right) ; 168.2$ $\left(\mathrm{C}=\mathrm{O}_{\text {carboxyl }}\right)$. MS (ESI): $m / z=318(M+\mathrm{H})^{+}$.

\section{Refinement}

Crystal data, data collection and structure refinement details are summarized in Table $4 . \mathrm{H}$ atoms attached to carbon were placed in calculated positions $(\mathrm{C}-\mathrm{H}=0.95-1.00 \AA)$, and were included as riding contributions with isotropic displacement parameters 1.2 or 1.5 times those of the attached atoms. Two reflections affected by the beamstop were omitted from the final refinement.

\section{Acknowledgements}

JTM thanks Tulane University for support of the Tulane Crystallography Laboratory. Authors' contributions are as follows. Conceptualization, YB; methodology, YB and NHA; investigation, $\mathrm{KC}$; theoretical calculations, $\mathrm{KC}$; writing (original draft), $\mathrm{KC}$; writing (review and editing of the manuscript), FHAO; supervision, EME; crystal-structure determination and validation, JTM.

\section{References}

Banu, S., Bollu, R., Bantu, R., Nagarapu, L., Polepalli, S., Jain, N., Vangala, R. \& Manga, V. (2017). Eur. J. Med. Chem. 125, 400410.

Becke, A. D. (1993). J. Chem. Phys. 98, 5648-5652.

Bouzian, Y., Karrouchi, K., Sert, Y., Lai, C.-H., Mahi, L., Ahabchane, N. H., Talbaoui, A., Mague, J. T. \& Essassi, E. M. (2020). J. Mol. Struct. 1209, 127940.

Brandenburg, K. \& Putz, H. (2012). DIAMOND, Crystal Impact GbR, Bonn, Germany.

Bruker (2020). APEX3 and SAINT. Bruker AXS LLC, Madison, Wisconsin, USA.

Chkirate, K., Azgaou, K., Elmsellem, H., El Ibrahimi, B., Sebbar, N. K., Anouar, E. H., Benmessaoud, M., El Hajjaji, S. \& Essassi, E. M. (2021). J. Mol. Liq. 321, 114750.

Chkirate, K., Fettach, S., El Hafi, M., Karrouchi, K., Elotmani, B., Mague, J. T., Radi, S., Faouzi, M. E. A., Adarsh, N. N., Essassi, E. M. \& Garcia, Y. (2020a). J. Inorg. Biochem. 208, 21-28.

Chkirate, K., Fettach, S., Karrouchi, K., Sebbar, N. K., Essassi, E. M., Mague, J. T., Radi, S., Faouzi, M. E. A., Adarsh, N. N. \& Garcia, Y. (2019). J. Inorg. Biochem. 191, 21-28.

Chkirate, K., Karrouchi, K., Dege, N., Sebbar, N. K., Ejjoummany, A., Radi, S., Adarsh, N. N., Talbaoui, A., Ferbinteanu, M., Essassi, E. M. \& Garcia, Y. (2020b). New J. Chem. 44, 2210-2221.

Fang, Y., Wu, Z., Xiao, M., Wei, L., Li, K., Tang, Y., Ye, J., Xiang, J. \& Hu, A. (2021). Bioorg. Chem. 106, 104469.

Filali Baba, Y., Misbahi, H., Kandri Rodi, Y., Ouzidan, Y., Essassi, E. M., Vincze, K., Nové, M., Gajdács, M., Molnár, J., Spengler, G. \& Mazzah, A. (2020). Chem. Data Collect. 29, 100501.

Filali Baba, Y., Sert, Y., Kandri Rodi, Y., Hayani, S., Mague, J. T., Prim, D., Marrot, J., Ouazzani Chahdi, F., Sebbar, N. K. \& Essassi, E. M. (2019). J. Mol. Struct. 1188, 255-268.

Frisch, M. J., Trucks, G. W., Schlegel, H. B., Scuseria, G. E., Robb, M. A., Cheeseman, J. R., Scalmani, G., Barone, V., Mennucci, B., Petersson, G. A., Nakatsuji, H., Caricato, M., Li, X., Hratchian, H. P., Izmaylov, A. F., Bloino, J., Zheng, G., Sonnenberg, J. L., Hada, M., Ehara, M., Toyota, K., Fukuda, R., Hasegawa, J., Ishida, M., Nakajima, T., Honda, Y., Kitao, O., Nakai, H., Vreven, T., Montgomery, J. A. Jr, Peralta, J. E., Ogliaro, F., Bearpark, M., Heyd, J. J., Brothers, E., Kudin, K. N., Staroverov, V. N., Kobayashi, R., Normand, J., Raghavachari, K., Rendell, A., Burant, J. C., Iyengar, S. S., Tomasi, J., Cossi, M., Rega, N., Millam, J. M., Klene, M., Knox, J. E., Cross, J. B., Bakken, V., Adamo, C., Jaramillo, J., Gomperts, R., Stratmann, R. E., Yazyev, O., Austin, A. J., Cammi, R., Pomelli, C., Ochterski, J. W., Martin, R. L., Morokuma, K., Zakrzewski, V. G., Voth, G. A., Salvador, P., Dannenberg, J. J., Dapprich, S., Daniels, A. D., Farkas, O., Foresman, J. B., Ortiz, J. V., Cioslowski, J. \& Fox, D. J. (2009). Gaussian 09. Revision A. 02. Gaussian Inc, Wallingford, CT, US.

Groom, C. R., Bruno, I. J., Lightfoot, M. P. \& Ward, S. C. (2016). Acta Cryst. B72, 171-179.

Hayani, S., Sert, Y., Filali Baba, Y., Benhiba, F., Ouazzani Chahdi, F., Laraqui, F.-Z., Mague, J. T., El Ibrahimi, B., Sebbar, N. K., Kandri Rodi, Y. \& Essassi, E. M. (2021). J. Mol. Struct. 1227, 129520.

Krause, L., Herbst-Irmer, R., Sheldrick, G. M. \& Stalke, D. (2015). J. Appl. Cryst. 48, 3-10.

Lee, C., Yang, W. \& Parr, R. G. (1988). Phys. Rev. B, 37, 785-789.

McKinnon, J. J., Jayatilaka, D. \& Spackman, M. A. (2007). Chem. Commun. pp. 3814-3816.

Sheldrick, G. M. (2008). Acta Cryst. A64, 112-122.

Sheldrick, G. M. (2015a). Acta Cryst. A71, 3-8.

Sheldrick, G. M. (2015b). Acta Cryst. C71, 3-8.

Turner, M. J., McKinnon, J. J., Wolff, S. K., Grimwood, D. J., Spackman, P. R., Jayatilaka, D. \& Spackman, M. A. (2017). CrystalExplorer17. The University of Western Australia. 


\section{supporting information}

Acta Cryst. (2021). E77, 824-828 [https://doi.org/10.1107/S2056989021007416]

\section{Crystal structure, Hirshfeld surface analysis and density functional theory study} of benzyl 2-oxo-1-(prop-2-yn-1-yl)-1,2-dihydroquinoline-4-carboxylate

\section{Younos Bouzian, Karim Chkirate, Joel T. Mague, Fares Hezam Al-Ostoot, Noureddine}

\section{Hammou Ahabchane and El Mokhtar Essassi}

\section{Computing details}

Data collection: APEX3 (Bruker, 2020); cell refinement: SAINT (Bruker, 2020); data reduction: SAINT (Bruker, 2020); program(s) used to solve structure: SHELXT (Sheldrick, 2015a); program(s) used to refine structure: SHELXL2018/1 (Sheldrick, 2015b); molecular graphics: DIAMOND (Brandenburg \& Putz, 2012); software used to prepare material for publication: SHELXTL (Sheldrick, 2008).

Benzyl 2-oxo-1-(prop-2-yn-1-yl)-1,2-dihydroquinoline-4-carboxylate

Crystal data

$\mathrm{C}_{20} \mathrm{H}_{15} \mathrm{NO}_{3}$

$M_{r}=317.33$

Monoclinic, $P 2{ }_{1} / n$

$a=8.2284(3) \AA$

$b=13.7693(4) \AA$

$c=13.9230(4) \AA$

$\beta=96.155(1)^{\circ}$

$V=1568.37(9) \AA^{3}$

$Z=4$

\section{Data collection}

Bruker D8 QUEST PHOTON 3 diffractometer

Radiation source: fine-focus sealed tube Graphite monochromator

Detector resolution: 7.3910 pixels $\mathrm{mm}^{-1}$ $\varphi$ and $\omega$ scans

Absorption correction: numerical (SADABS; Krause et al., 2015)

$T_{\min }=0.93, T_{\max }=0.97$

\section{Refinement}

Refinement on $F^{2}$

Least-squares matrix: full

$R\left[F^{2}>2 \sigma\left(F^{2}\right)\right]=0.046$

$w R\left(F^{2}\right)=0.133$

$S=1.03$

6020 reflections

217 parameters

0 restraints
$F(000)=664$

$D_{\mathrm{x}}=1.344 \mathrm{Mg} \mathrm{m}^{-3}$

Mo $K \alpha$ radiation, $\lambda=0.71073 \AA$

Cell parameters from 9939 reflections

$\theta=2.5-33.3^{\circ}$

$\mu=0.09 \mathrm{~mm}^{-1}$

$T=150 \mathrm{~K}$

Block, colourless

$0.44 \times 0.35 \times 0.32 \mathrm{~mm}$

80207 measured reflections

6020 independent reflections

5304 reflections with $I>2 \sigma(I)$

$R_{\text {int }}=0.025$

$\theta_{\max }=33.4^{\circ}, \theta_{\min }=2.9^{\circ}$

$h=-12 \rightarrow 12$

$k=-21 \rightarrow 21$

$l=-21 \rightarrow 21$

Primary atom site location: dual

Secondary atom site location: difference Fourier map

Hydrogen site location: inferred from neighbouring sites

$\mathrm{H}$-atom parameters constrained

$w=1 /\left[\sigma^{2}\left(F_{\mathrm{o}}^{2}\right)+(0.0739 P)^{2}+0.3685 P\right]$

where $P=\left(F_{\mathrm{o}}{ }^{2}+2 F_{\mathrm{c}}{ }^{2}\right) / 3$ 
$(\Delta / \sigma)_{\max }=0.001$

$\Delta \rho_{\max }=0.46$ e $\AA^{-3}$

Special details

Experimental. The diffraction data were obtained from 9 sets of frames, each of width $0.5^{\circ}$ in $\omega$ or $\varphi$, collected with scan parameters determined by the "strategy" routine in APEX3. The scan time was $7 \mathrm{sec} /$ frame.

Geometry. All esds (except the esd in the dihedral angle between two 1.s. planes) are estimated using the full covariance matrix. The cell esds are taken into account individually in the estimation of esds in distances, angles and torsion angles; correlations between esds in cell parameters are only used when they are defined by crystal symmetry. An approximate (isotropic) treatment of cell esds is used for estimating esds involving l.s. planes.

Refinement. Refinement of $\mathrm{F}^{2}$ against ALL reflections. The weighted R-factor $\mathrm{wR}$ and goodness of fit $\mathrm{S}$ are based on $\mathrm{F}^{2}$, conventional $R$-factors $R$ are based on $F$, with $F$ set to zero for negative $F^{2}$. The threshold expression of $F^{2}>2 \operatorname{sigma}\left(\mathrm{F}^{2}\right)$ is used only for calculating R-factors(gt) etc. and is not relevant to the choice of reflections for refinement. R-factors based on $\mathrm{F}^{2}$ are statistically about twice as large as those based on F, and R- factors based on ALL data will be even larger. $\mathrm{H}$-atoms attached to carbon were placed in calculated positions $(\mathrm{C}-\mathrm{H}=0.95-1.00 \AA)$. All were included as riding contributions with isotropic displacement parameters 1.2 - 1.5 times those of the attached atoms. Two reflections affected by the beamstop were omitted from the final refinement.

Fractional atomic coordinates and isotropic or equivalent isotropic displacement parameters $\left(\AA^{2}\right)$

\begin{tabular}{|c|c|c|c|c|}
\hline & $x$ & $y$ & $z$ & $U_{\text {iso }} * / U_{\text {eq }}$ \\
\hline $\mathrm{O} 1$ & $0.79130(8)$ & $0.56932(5)$ & $0.29079(6)$ & $0.03386(17)$ \\
\hline $\mathrm{O} 2$ & $0.15346(8)$ & $0.39853(5)$ & $0.36012(6)$ & $0.03159(16)$ \\
\hline $\mathrm{O} 3$ & $0.34027(8)$ & $0.35057(5)$ & $0.26450(5)$ & $0.02547(14)$ \\
\hline N1 & $0.63622(8)$ & $0.63640(5)$ & $0.39987(5)$ & $0.01811(12)$ \\
\hline $\mathrm{C} 1$ & $0.49088(9)$ & $0.64053(5)$ & $0.44357(5)$ & $0.01656(13)$ \\
\hline $\mathrm{C} 2$ & $0.46483(10)$ & $0.71713(6)$ & $0.50694(6)$ & $0.02098(14)$ \\
\hline $\mathrm{H} 2$ & 0.544815 & 0.766658 & 0.518906 & $0.025^{*}$ \\
\hline $\mathrm{C} 3$ & $0.32280(11)$ & $0.72050(6)$ & $0.55192(6)$ & $0.02469(16)$ \\
\hline H3 & 0.306866 & 0.771800 & 0.595567 & $0.030 *$ \\
\hline $\mathrm{C} 4$ & $0.20287(10)$ & $0.64925(7)$ & $0.53375(6)$ & $0.02508(16)$ \\
\hline $\mathrm{H} 4$ & 0.106080 & 0.651845 & 0.565229 & $0.030 *$ \\
\hline $\mathrm{C} 5$ & $0.22538(10)$ & $0.57466(6)$ & $0.46961(6)$ & $0.02108(14)$ \\
\hline H5 & 0.142234 & 0.527154 & 0.456509 & $0.025^{*}$ \\
\hline C6 & $0.36952(9)$ & $0.56798(5)$ & $0.42340(5)$ & $0.01629(13)$ \\
\hline $\mathrm{C} 7$ & $0.40167(8)$ & $0.49162(5)$ & $0.35579(5)$ & $0.01659(13)$ \\
\hline $\mathrm{C} 8$ & $0.54184(9)$ & $0.49270(6)$ & $0.31341(6)$ & $0.02043(14)$ \\
\hline H8 & 0.560136 & 0.442273 & 0.269253 & $0.025^{*}$ \\
\hline $\mathrm{C} 9$ & $0.66584(10)$ & $0.56733(6)$ & $0.33219(6)$ & $0.02148(15)$ \\
\hline $\mathrm{C} 10$ & $0.76472(10)$ & $0.71018(6)$ & $0.42091(6)$ & $0.02241(15)$ \\
\hline $\mathrm{H} 10 \mathrm{~A}$ & 0.775440 & 0.725750 & 0.490720 & $0.027 *$ \\
\hline H10B & 0.870536 & 0.683646 & 0.405222 & $0.027 *$ \\
\hline C11 & $0.72727(11)$ & $0.79935(6)$ & $0.36498(7)$ & $0.02598(17)$ \\
\hline $\mathrm{C} 12$ & $0.69298(13)$ & $0.87027(8)$ & $0.31880(9)$ & $0.0356(2)$ \\
\hline H12 & 0.665529 & 0.927061 & 0.281827 & $0.043 *$ \\
\hline $\mathrm{C} 13$ & $0.28317(9)$ & $0.41024(5)$ & $0.32886(6)$ & $0.01886(14)$ \\
\hline C14 & $0.23208(11)$ & $0.27253(7)$ & $0.22635(6)$ & $0.02652(17)$ \\
\hline H14A & 0.266692 & 0.250123 & 0.164117 & $0.032 *$ \\
\hline H14B & 0.119369 & 0.298095 & 0.213628 & $0.032 *$ \\
\hline
\end{tabular}




\begin{tabular}{lllll} 
C15 & $0.23223(10)$ & $0.18782(6)$ & $0.29404(6)$ & $0.02316(15)$ \\
C16 & $0.36596(12)$ & $0.12502(8)$ & $0.30625(7)$ & $0.0318(2)$ \\
H16 & 0.457268 & 0.135594 & 0.271197 & $0.038^{*}$ \\
C17 & $0.36579(17)$ & $0.04679(9)$ & $0.36979(9)$ & $0.0430(3)$ \\
H17 & 0.457907 & 0.004905 & 0.378888 & $0.052^{*}$ \\
C18 & $0.2320(2)$ & $0.02998(9)$ & $0.41959(9)$ & $0.0502(3)$ \\
H18 & 0.232480 & -0.023368 & 0.462895 & $0.060^{*}$ \\
C19 & $0.09713(18)$ & $0.09064(9)$ & $0.40660(9)$ & $0.0442(3)$ \\
H19 & 0.004674 & 0.078535 & 0.440243 & $0.053^{*}$ \\
C20 & $0.09774(12)$ & $0.16940(7)$ & $0.34402(7)$ & $0.02989(19)$ \\
H20 & 0.005332 & 0.211073 & 0.335298 & $0.036^{*}$ \\
\hline
\end{tabular}

Atomic displacement parameters $\left(\AA^{2}\right)$

\begin{tabular}{lllllll}
\hline & $U^{11}$ & $U^{22}$ & $U^{33}$ & $U^{12}$ & $U^{13}$ & $U^{23}$ \\
\hline O1 & $0.0257(3)$ & $0.0298(3)$ & $0.0496(4)$ & $-0.0088(3)$ & $0.0203(3)$ & $-0.0130(3)$ \\
O2 & $0.0222(3)$ & $0.0270(3)$ & $0.0474(4)$ & $-0.0083(2)$ & $0.0127(3)$ & $-0.0108(3)$ \\
O3 & $0.0263(3)$ & $0.0234(3)$ & $0.0276(3)$ & $-0.0089(2)$ & $0.0067(2)$ & $-0.0090(2)$ \\
N1 & $0.0171(3)$ & $0.0150(3)$ & $0.0224(3)$ & $-0.0026(2)$ & $0.0026(2)$ & $-0.0004(2)$ \\
C1 & $0.0176(3)$ & $0.0148(3)$ & $0.0171(3)$ & $0.0000(2)$ & $0.0009(2)$ & $0.0012(2)$ \\
C2 & $0.0242(3)$ & $0.0177(3)$ & $0.0208(3)$ & $-0.0006(3)$ & $0.0015(3)$ & $-0.0023(2)$ \\
C3 & $0.0270(4)$ & $0.0233(4)$ & $0.0240(3)$ & $0.0028(3)$ & $0.0041(3)$ & $-0.0051(3)$ \\
C4 & $0.0219(3)$ & $0.0277(4)$ & $0.0265(4)$ & $0.0025(3)$ & $0.0070(3)$ & $-0.0035(3)$ \\
C5 & $0.0182(3)$ & $0.0223(3)$ & $0.0232(3)$ & $0.0000(2)$ & $0.0040(2)$ & $-0.0010(3)$ \\
C6 & $0.0160(3)$ & $0.0154(3)$ & $0.0174(3)$ & $0.0005(2)$ & $0.0011(2)$ & $0.0012(2)$ \\
C7 & $0.0165(3)$ & $0.0145(3)$ & $0.0186(3)$ & $-0.0011(2)$ & $0.0013(2)$ & $0.0007(2)$ \\
C8 & $0.0196(3)$ & $0.0164(3)$ & $0.0260(3)$ & $-0.0028(2)$ & $0.0059(3)$ & $-0.0032(2)$ \\
C9 & $0.0191(3)$ & $0.0178(3)$ & $0.0284(4)$ & $-0.0025(2)$ & $0.0068(3)$ & $-0.0027(3)$ \\
C10 & $0.0197(3)$ & $0.0192(3)$ & $0.0281(4)$ & $-0.0051(3)$ & $0.0013(3)$ & $-0.0009(3)$ \\
C11 & $0.0237(3)$ & $0.0219(4)$ & $0.0332(4)$ & $-0.0062(3)$ & $0.0072(3)$ & $0.0002(3)$ \\
C12 & $0.0313(4)$ & $0.0291(4)$ & $0.0484(6)$ & $-0.0021(4)$ & $0.0138(4)$ & $0.0106(4)$ \\
C13 & $0.0183(3)$ & $0.0167(3)$ & $0.0213(3)$ & $-0.0018(2)$ & $0.0008(2)$ & $-0.0001(2)$ \\
C14 & $0.0295(4)$ & $0.0249(4)$ & $0.0247(4)$ & $-0.0092(3)$ & $0.0008(3)$ & $-0.0067(3)$ \\
C15 & $0.0230(3)$ & $0.0217(3)$ & $0.0250(3)$ & $-0.0049(3)$ & $0.0034(3)$ & $-0.0082(3)$ \\
C16 & $0.0268(4)$ & $0.0337(5)$ & $0.0345(4)$ & $0.0020(3)$ & $0.0016(3)$ & $-0.0126(4)$ \\
C17 & $0.0522(7)$ & $0.0316(5)$ & $0.0426(6)$ & $0.0117(5)$ & $-0.0067(5)$ & $-0.0085(4)$ \\
C18 & $0.0824(10)$ & $0.0299(5)$ & $0.0385(6)$ & $-0.0013(6)$ & $0.0066(6)$ & $0.0026(4)$ \\
C19 & $0.0619(7)$ & $0.0333(5)$ & $0.0409(6)$ & $-0.0112(5)$ & $0.0214(5)$ & $-0.0035(4)$ \\
C20 & $0.0309(4)$ & $0.0253(4)$ & $0.0355(4)$ & $-0.0054(3)$ & $0.0123(3)$ & $-0.0084(3)$ \\
& & & & & &
\end{tabular}

Geometric parameters $\left(\AA,{ }^{\circ}\right)$

\begin{tabular}{llll}
\hline $\mathrm{O} 1-\mathrm{C} 9$ & $1.2355(10)$ & $\mathrm{C} 8-\mathrm{H} 8$ & 0.9500 \\
$\mathrm{O} 2-\mathrm{C} 13$ & $1.2058(10)$ & $\mathrm{C} 10-\mathrm{C} 11$ & $1.4687(12)$ \\
$\mathrm{O} 3-\mathrm{C} 13$ & $1.3375(10)$ & $\mathrm{C} 10-\mathrm{H} 10 \mathrm{~A}$ & 0.9900 \\
$\mathrm{O} 3-\mathrm{C} 14$ & $1.4588(10)$ & $\mathrm{C} 10-\mathrm{H} 10 \mathrm{~B}$ & 0.9900 \\
$\mathrm{~N} 1-\mathrm{C} 9$ & $1.3788(10)$ & $\mathrm{C} 11-\mathrm{C} 12$ & $1.1865(14)$ \\
$\mathrm{N} 1-\mathrm{C} 1$ & $1.3999(10)$ & $\mathrm{C} 12-\mathrm{H} 12$ & 0.9500
\end{tabular}




\begin{tabular}{|c|c|c|c|}
\hline $\mathrm{N} 1-\mathrm{C} 10$ & $1.4730(10)$ & $\mathrm{C} 14-\mathrm{C} 15$ & $1.4995(13)$ \\
\hline $\mathrm{C} 1-\mathrm{C} 2$ & $1.4062(10)$ & $\mathrm{C} 14-\mathrm{H} 14 \mathrm{~A}$ & 0.9900 \\
\hline $\mathrm{C} 1-\mathrm{C} 6$ & $1.4192(10)$ & C14-H14B & 0.9900 \\
\hline $\mathrm{C} 2-\mathrm{C} 3$ & $1.3846(11)$ & $\mathrm{C} 15-\mathrm{C} 20$ & $1.3922(12)$ \\
\hline $\mathrm{C} 2-\mathrm{H} 2$ & 0.9500 & $\mathrm{C} 15-\mathrm{C} 16$ & $1.3955(13)$ \\
\hline $\mathrm{C} 3-\mathrm{C} 4$ & $1.3953(12)$ & $\mathrm{C} 16-\mathrm{C} 17$ & $1.3940(17)$ \\
\hline $\mathrm{C} 3-\mathrm{H} 3$ & 0.9500 & $\mathrm{C} 16-\mathrm{H} 16$ & 0.9500 \\
\hline $\mathrm{C} 4-\mathrm{C} 5$ & $1.3864(11)$ & $\mathrm{C} 17-\mathrm{C} 18$ & $1.382(2)$ \\
\hline $\mathrm{C} 4-\mathrm{H} 4$ & 0.9500 & $\mathrm{C} 17-\mathrm{H} 17$ & 0.9500 \\
\hline $\mathrm{C} 5-\mathrm{C} 6$ & $1.4116(10)$ & $\mathrm{C} 18-\mathrm{C} 19$ & $1.385(2)$ \\
\hline $\mathrm{C} 5-\mathrm{H} 5$ & 0.9500 & $\mathrm{C} 18-\mathrm{H} 18$ & 0.9500 \\
\hline $\mathrm{C} 6-\mathrm{C} 7$ & $1.4543(10)$ & $\mathrm{C} 19-\mathrm{C} 20$ & $1.3914(16)$ \\
\hline $\mathrm{C} 7-\mathrm{C} 8$ & $1.3507(10)$ & C19-H19 & 0.9500 \\
\hline $\mathrm{C} 7-\mathrm{C} 13$ & $1.5062(10)$ & $\mathrm{C} 20-\mathrm{H} 20$ & 0.9500 \\
\hline $\mathrm{C} 8-\mathrm{C} 9$ & $1.4520(11)$ & & \\
\hline $\mathrm{C} 13-\mathrm{O} 3-\mathrm{C} 14$ & $116.87(7)$ & $\mathrm{N} 1-\mathrm{C} 10-\mathrm{H} 10 \mathrm{~A}$ & 109.3 \\
\hline $\mathrm{C} 9-\mathrm{N} 1-\mathrm{C} 1$ & $123.16(6)$ & $\mathrm{C} 11-\mathrm{C} 10-\mathrm{H} 10 \mathrm{~B}$ & 109.3 \\
\hline $\mathrm{C} 9-\mathrm{N} 1-\mathrm{C} 10$ & $115.85(6)$ & $\mathrm{N} 1-\mathrm{C} 10-\mathrm{H} 10 \mathrm{~B}$ & 109.3 \\
\hline $\mathrm{C} 1-\mathrm{N} 1-\mathrm{C} 10$ & $120.93(6)$ & $\mathrm{H} 10 \mathrm{~A}-\mathrm{C} 10-\mathrm{H} 10 \mathrm{~B}$ & 108.0 \\
\hline $\mathrm{N} 1-\mathrm{C} 1-\mathrm{C} 2$ & $119.87(7)$ & $\mathrm{C} 12-\mathrm{C} 11-\mathrm{C} 10$ & $178.18(10)$ \\
\hline $\mathrm{N} 1-\mathrm{C} 1-\mathrm{C} 6$ & $120.08(6)$ & $\mathrm{C} 11-\mathrm{C} 12-\mathrm{H} 12$ & 180.0 \\
\hline $\mathrm{C} 2-\mathrm{C} 1-\mathrm{C} 6$ & $120.05(7)$ & $\mathrm{O} 2-\mathrm{C} 13-\mathrm{O} 3$ & $123.21(7)$ \\
\hline $\mathrm{C} 3-\mathrm{C} 2-\mathrm{C} 1$ & $120.12(7)$ & $\mathrm{O} 2-\mathrm{C} 13-\mathrm{C} 7$ & $125.74(7)$ \\
\hline $\mathrm{C} 3-\mathrm{C} 2-\mathrm{H} 2$ & 119.9 & $\mathrm{O} 3-\mathrm{C} 13-\mathrm{C} 7$ & $111.05(6)$ \\
\hline $\mathrm{C} 1-\mathrm{C} 2-\mathrm{H} 2$ & 119.9 & $\mathrm{O} 3-\mathrm{C} 14-\mathrm{C} 15$ & $112.63(7)$ \\
\hline $\mathrm{C} 2-\mathrm{C} 3-\mathrm{C} 4$ & $120.62(7)$ & $\mathrm{O} 3-\mathrm{C} 14-\mathrm{H} 14 \mathrm{~A}$ & 109.1 \\
\hline $\mathrm{C} 2-\mathrm{C} 3-\mathrm{H} 3$ & 119.7 & $\mathrm{C} 15-\mathrm{C} 14-\mathrm{H} 14 \mathrm{~A}$ & 109.1 \\
\hline $\mathrm{C} 4-\mathrm{C} 3-\mathrm{H} 3$ & 119.7 & $\mathrm{O} 3-\mathrm{C} 14-\mathrm{H} 14 \mathrm{~B}$ & 109.1 \\
\hline $\mathrm{C} 5-\mathrm{C} 4-\mathrm{C} 3$ & $119.80(8)$ & $\mathrm{C} 15-\mathrm{C} 14-\mathrm{H} 14 \mathrm{~B}$ & 109.1 \\
\hline $\mathrm{C} 5-\mathrm{C} 4-\mathrm{H} 4$ & 120.1 & $\mathrm{H} 14 \mathrm{~A}-\mathrm{C} 14-\mathrm{H} 14 \mathrm{~B}$ & 107.8 \\
\hline $\mathrm{C} 3-\mathrm{C} 4-\mathrm{H} 4$ & 120.1 & $\mathrm{C} 20-\mathrm{C} 15-\mathrm{C} 16$ & $119.00(9)$ \\
\hline $\mathrm{C} 4-\mathrm{C} 5-\mathrm{C} 6$ & $121.24(7)$ & $\mathrm{C} 20-\mathrm{C} 15-\mathrm{C} 14$ & $120.58(8)$ \\
\hline $\mathrm{C} 4-\mathrm{C} 5-\mathrm{H} 5$ & 119.4 & $\mathrm{C} 16-\mathrm{C} 15-\mathrm{C} 14$ & $120.41(8)$ \\
\hline $\mathrm{C} 6-\mathrm{C} 5-\mathrm{H} 5$ & 119.4 & $\mathrm{C} 17-\mathrm{C} 16-\mathrm{C} 15$ & $120.11(10)$ \\
\hline $\mathrm{C} 5-\mathrm{C} 6-\mathrm{C} 1$ & $118.15(7)$ & $\mathrm{C} 17-\mathrm{C} 16-\mathrm{H} 16$ & 119.9 \\
\hline $\mathrm{C} 5-\mathrm{C} 6-\mathrm{C} 7$ & $124.21(7)$ & $\mathrm{C} 15-\mathrm{C} 16-\mathrm{H} 16$ & 119.9 \\
\hline $\mathrm{C} 1-\mathrm{C} 6-\mathrm{C} 7$ & $117.65(6)$ & $\mathrm{C} 18-\mathrm{C} 17-\mathrm{C} 16$ & $120.18(11)$ \\
\hline $\mathrm{C} 8-\mathrm{C} 7-\mathrm{C} 6$ & $119.87(6)$ & $\mathrm{C} 18-\mathrm{C} 17-\mathrm{H} 17$ & 119.9 \\
\hline $\mathrm{C} 8-\mathrm{C} 7-\mathrm{C} 13$ & $117.37(7)$ & $\mathrm{C} 16-\mathrm{C} 17-\mathrm{H} 17$ & 119.9 \\
\hline $\mathrm{C} 6-\mathrm{C} 7-\mathrm{C} 13$ & $122.76(6)$ & $\mathrm{C} 17-\mathrm{C} 18-\mathrm{C} 19$ & $120.23(11)$ \\
\hline $\mathrm{C} 7-\mathrm{C} 8-\mathrm{C} 9$ & $123.06(7)$ & $\mathrm{C} 17-\mathrm{C} 18-\mathrm{H} 18$ & 119.9 \\
\hline $\mathrm{C} 7-\mathrm{C} 8-\mathrm{H} 8$ & 118.5 & $\mathrm{C} 19-\mathrm{C} 18-\mathrm{H} 18$ & 119.9 \\
\hline $\mathrm{C} 9-\mathrm{C} 8-\mathrm{H} 8$ & 118.5 & $\mathrm{C} 18-\mathrm{C} 19-\mathrm{C} 20$ & $119.68(11)$ \\
\hline $\mathrm{O} 1-\mathrm{C} 9-\mathrm{N} 1$ & $121.42(7)$ & $\mathrm{C} 18-\mathrm{C} 19-\mathrm{H} 19$ & 120.2 \\
\hline $\mathrm{O} 1-\mathrm{C} 9-\mathrm{C} 8$ & $122.54(7)$ & $\mathrm{C} 20-\mathrm{C} 19-\mathrm{H} 19$ & 120.2 \\
\hline $\mathrm{N} 1-\mathrm{C} 9-\mathrm{C} 8$ & $116.04(7)$ & $\mathrm{C} 19-\mathrm{C} 20-\mathrm{C} 15$ & $120.76(10)$ \\
\hline $\mathrm{C} 11-\mathrm{C} 10-\mathrm{N} 1$ & $111.46(7)$ & $\mathrm{C} 19-\mathrm{C} 20-\mathrm{H} 20$ & 119.6 \\
\hline
\end{tabular}




$\begin{array}{llll}\mathrm{C} 11-\mathrm{C} 10-\mathrm{H} 10 \mathrm{~A} & 109.3 & \mathrm{C} 15-\mathrm{C} 20-\mathrm{H} 20 & 119.6 \\ \mathrm{C} 9-\mathrm{N} 1-\mathrm{C} 1-\mathrm{C} 2 & -175.56(7) & \mathrm{C} 1-\mathrm{N} 1-\mathrm{C} 9-\mathrm{C} 8 & -4.77(11) \\ \mathrm{C} 10-\mathrm{N} 1-\mathrm{C} 1-\mathrm{C} 2 & 1.37(10) & \mathrm{C} 10-\mathrm{N} 1-\mathrm{C} 9-\mathrm{C} 8 & 178.16(7) \\ \mathrm{C} 9-\mathrm{N} 1-\mathrm{C} 1-\mathrm{C} 6 & 3.97(11) & \mathrm{C} 7-\mathrm{C} 8-\mathrm{C} 9-\mathrm{O} 1 & -177.70(9) \\ \mathrm{C} 10-\mathrm{N} 1-\mathrm{C} 1-\mathrm{C} 6 & -179.10(7) & \mathrm{C} 7-\mathrm{C} 8-\mathrm{C} 9-\mathrm{N} 1 & 2.64(12) \\ \mathrm{N} 1-\mathrm{C} 1-\mathrm{C} 2-\mathrm{C} 3 & -178.65(7) & \mathrm{C} 9-\mathrm{N} 1-\mathrm{C} 10-\mathrm{C} 11 & 96.66(8) \\ \mathrm{C} 6-\mathrm{C} 1-\mathrm{C} 2-\mathrm{C} 3 & 1.82(11) & \mathrm{C} 1-\mathrm{N} 1-\mathrm{C} 10-\mathrm{C} 11 & -80.48(9) \\ \mathrm{C} 1-\mathrm{C} 2-\mathrm{C} 3-\mathrm{C} 4 & -1.16(13) & \mathrm{C} 14-\mathrm{O} 3-\mathrm{C} 13-\mathrm{O} 2 & 4.71(12) \\ \mathrm{C} 2-\mathrm{C} 3-\mathrm{C} 4-\mathrm{C} 5 & -0.41(13) & \mathrm{C} 14-\mathrm{O} 3-\mathrm{C} 13-\mathrm{C} 7 & -175.41(7) \\ \mathrm{C} 3-\mathrm{C} 4-\mathrm{C} 5-\mathrm{C} 6 & 1.33(13) & \mathrm{C} 8-\mathrm{C} 7-\mathrm{C} 13-\mathrm{O} 2 & 179.78(8) \\ \mathrm{C} 4-\mathrm{C} 5-\mathrm{C} 6-\mathrm{C} 1 & -0.66(11) & \mathrm{C} 6-\mathrm{C} 7-\mathrm{C} 13-\mathrm{O} 2 & -1.07(12) \\ \mathrm{C} 4-\mathrm{C} 5-\mathrm{C} 6-\mathrm{C} 7 & 179.70(7) & \mathrm{C} 8-\mathrm{C} 7-\mathrm{C} 13-\mathrm{O} 3 & -0.10(10) \\ \mathrm{N} 1-\mathrm{C} 1-\mathrm{C} 6-\mathrm{C} 5 & 179.56(7) & \mathrm{C} 6-\mathrm{C} 7-\mathrm{C} 13-\mathrm{O} 3 & 179.05(7) \\ \mathrm{C} 2-\mathrm{C} 1-\mathrm{C} 6-\mathrm{C} 5 & -0.91(11) & \mathrm{C} 13-\mathrm{O} 3-\mathrm{C} 14-\mathrm{C} 15 & -80.18(10) \\ \mathrm{N} 1-\mathrm{C} 1-\mathrm{C} 6-\mathrm{C} 7 & -0.77(10) & \mathrm{O} 3-\mathrm{C} 14-\mathrm{C} 15-\mathrm{C} 20 & 107.82(9) \\ \mathrm{C} 2-\mathrm{C} 1-\mathrm{C} 6-\mathrm{C} 7 & 178.76(7) & \mathrm{O} 3-\mathrm{C} 14-\mathrm{C} 15-\mathrm{C} 16 & -73.57(10) \\ \mathrm{C} 5-\mathrm{C} 6-\mathrm{C} 7-\mathrm{C} 8 & 178.41(7) & \mathrm{C} 20-\mathrm{C} 15-\mathrm{C} 16-\mathrm{C} 17 & -1.82(13) \\ \mathrm{C} 1-\mathrm{C} 6-\mathrm{C} 7-\mathrm{C} 8 & -1.23(11) & \mathrm{C} 14-\mathrm{C} 15-\mathrm{C} 16-\mathrm{C} 17 & 179.56(8) \\ \mathrm{C} 5-\mathrm{C} 6-\mathrm{C} 7-\mathrm{C} 13 & -0.72(11) & \mathrm{C} 15-\mathrm{C} 16-\mathrm{C} 17-\mathrm{C} 18 & 1.23(16) \\ \mathrm{C} 1-\mathrm{C} 6-\mathrm{C} 7-\mathrm{C} 13 & 179.64(6) & \mathrm{C} 16-\mathrm{C} 17-\mathrm{C} 18-\mathrm{C} 19 & 0.10(18) \\ \mathrm{C} 6-\mathrm{C} 7-\mathrm{C} 8-\mathrm{C} 9 & 0.28(12) & \mathrm{C} 17-\mathrm{C} 18-\mathrm{C} 19-\mathrm{C} 20 & -0.80(19) \\ \mathrm{C} 13-\mathrm{C} 7-\mathrm{C} 8-\mathrm{C} 9 & 179.45(7) & \mathrm{C} 18-\mathrm{C} 19-\mathrm{C} 20-\mathrm{C} 15 & 0.19(17) \\ \mathrm{C} 1-\mathrm{N} 1-\mathrm{C} 9-\mathrm{O} 1 & 175.57(8) & \mathrm{C} 16-\mathrm{C} 15-\mathrm{C} 20-\mathrm{C} 19 & 1.12(14) \\ \mathrm{C} 10-\mathrm{N} 1-\mathrm{C} 9-\mathrm{O} 1 & -1.50(12) & \mathrm{C} 14-\mathrm{C} 15-\mathrm{C} 20-\mathrm{C} 19 & 179.74(9)\end{array}$

Hydrogen-bond geometry $\left(A,{ }^{\circ}\right)$

$\mathrm{Cg} 2$ and $\mathrm{Cg} 3$ are the centroids of the $\mathrm{C} 1-\mathrm{C} 6$ and $\mathrm{C} 15-\mathrm{C} 20$ benzene rings, respectively.

\begin{tabular}{lllll}
\hline$D-\mathrm{H} \cdots A$ & $D-\mathrm{H}$ & $\mathrm{H} \cdots A$ & $D \cdots A$ & $D-\mathrm{H} \cdots A$ \\
\hline $\mathrm{C} 2-\mathrm{H} 2 \cdots C g 3^{\mathrm{i}}$ & 0.95 & 2.94 & $3.8206(10)$ & 154 \\
$\mathrm{C} 4-\mathrm{H} 4 \cdots \mathrm{O} 2^{\mathrm{ii}}$ & 0.95 & 2.57 & $3.4846(11)$ & 162 \\
$\mathrm{C} 5-\mathrm{H} 5 \cdots \mathrm{O} 2$ & 0.95 & 2.23 & $2.8917(11)$ & 126 \\
$\mathrm{C} 12-\mathrm{H} 12 \cdots \mathrm{O} 1^{\mathrm{iii}}$ & 0.95 & 2.25 & $3.1463(14)$ & 157 \\
$\mathrm{C} 14-\mathrm{H} 14 A \cdots C g 2^{\mathrm{iv}}$ & 0.99 & 2.65 & $3.4652(9)$ & 140 \\
$\mathrm{C} 16-\mathrm{H} 16 \cdots{ }^{v}$ & 0.95 & 2.50 & $3.3443(12)$ & 148
\end{tabular}

Symmetry codes: (i) $-x+1,-y+1,-z+1$; (ii) $-x,-y+1,-z+1$; (iii) $-x+3 / 2, y+1 / 2,-z+1 / 2$; (iv) $-x+1 / 2, y-1 / 2,-z+1 / 2$; (v) $-x+3 / 2, y-1 / 2,-z+1 / 2$. 